Research Article

\title{
Methicillin-Resistant Coagulase-Negative Staphylococci Carriage is a Protective Factor of Methicillin-Resistant Staphylococcus Aureus Nasal Colonization in HIV-Infected Patients: A Cross-Sectional Study
}

\author{
Ying Li ${ }^{1},{ }^{1}$ Jialing Lin, ${ }^{2}$ Linghua Li, ${ }^{3}$ Weiping Cai, ${ }^{3}$ Jiaping Ye, ${ }^{4}$ Suiping He, \\ Wencui Zhang, ${ }^{1}$ Ning Liu, ${ }^{1}$ Zijun Gong, ${ }^{1}$ Xiaohua Ye, ${ }^{1}$ and Zhenjiang Yao $\mathbb{D}{ }^{1}$ \\ ${ }^{1}$ Department of Epidemiology and Health Statistics, School of Public Health, Guangdong Pharmaceutical University, \\ Guangzhou, China \\ ${ }^{2}$ School of Population Health, The University of New South Wales, Sydney, Australia \\ ${ }^{3}$ Department of Infectious Diseases, Guangzhou Eighth People's Hospital, Guangzhou Medical University, Guangzhou, China \\ ${ }^{4}$ Department of Preventive Health Care, Beihai People's Hospital, Beihai, China
}

Correspondence should be addressed to Zhenjiang Yao; zhjyao2001@yahoo.com

Received 5 July 2020; Revised 10 September 2020; Accepted 26 December 2020; Published 12 January 2021

Academic Editor: José Ramón Blanco

Copyright (C) 2021 Ying Li et al. This is an open access article distributed under the Creative Commons Attribution License, which permits unrestricted use, distribution, and reproduction in any medium, provided the original work is properly cited.

\begin{abstract}
Background. Methicillin-resistant coagulase-negative Staphylococci (MRCoNS) is regarded as the repository of mecA gene for methicillin-resistant Staphylococcus aureus (MRSA) and may develop methicillin-susceptible Staphylococcus aureus (MSSA) to MRSA. Therefore, we aimed to explore whether MRCoNS carriage is a risk factor of MRSA colonization. Phenotypic characteristics were performed to further assess the associations between MRSA and MRCoNS. Methods. This cross-sectional study was conducted in Guangzhou, China. Participants completed a questionnaire and provided a nasal swab for further analysis. The risk factors of MRSA colonization were analyzed using nonconditional logistic regression models. The phenotypic characteristics between MRSA and MRCoNS were compared by Chi-square test. Results. Among the $1001 \mathrm{HIV}$-infected patients, a total of 119 (11.89\%) participants were positive for MRSA, and 34.45\% (41/119) of all MRSA carriers were positive for MRCoNS. We found MRCoNS carriage was a protective factor of MRSA colonization (adjusted odds ratio $=0.59,95 \%$ confidence interval: $0.38-0.91$ ). A significant difference in the proportions of antibiotic resistance between MRSA and MRCoNS isolates was found except for penicillin, clindamycin, tetracycline, and teicoplanin. The main STs and CC types of MRSA isolates in this population were ST188 (15.1\%) and CC59 (17.6\%), respectively. Conclusions. HIV-infected patients remain a highly vulnerable population for MRSA colonization. Though who carried MRCoNS is less likely to have MRSA colonization, similarity of some antibiotic resistance between MRSA and MRCoNS was found in this study. Regular surveillance on the colonization and antibiotic patterns of MRSA and MRCoNS is still necessary.
\end{abstract}

\section{Introduction}

As a common cause of severe infections, methicillin-resistant Staphylococcus aureus (MRSA) is still widespread in both health facilities and the community $[1,2]$. Owing to the compromised immune system, HIV-infected patients are more vulnerable to MRSA colonization than other populations $[3,4]$. Over the past decade, the prevalence of
MRSA isolates and the risk factors associated with MRSA colonization among HIV-infected patients has been reported in many countries and regions except mainland China. A systematic review reported that the pooled worldwide prevalence of MRSA in HIV-infected patients was 7\%, with the highest prevalence in Southeast Asia (16\%) and lowest prevalence in the European region (1\%) [5]. It was also found that prior use of antibiotics, recent CD4 
count less than 200, and prior hospitalization were general potential risk factors of MRSA colonization for persons who were HIV-positive [6,7].

Notably, a study suggested that methicillin-resistant coagulase-negative Staphylococci (MRCoNS) is a source of the mecA gene, the methicillin-resistance gene, and has the potential contribution to the emergence of MRSA [8]. It also reported that co-colonization of MRSA and MRCoNS isolates in humans may lead to horizontal cross-propagation of resistance genes [9]. In other words, there may be a relationship between MRSA and MRCoNS at molecular level. We therefore hypothesize that there is a possible link between them on population's level, which means MRCoNS carriage may be a potential risk factor of MRSA colonization in $\mathrm{HIV}$-positive patients. However, there is no relevant research on this relationship. Thus, we design this crosssectional study to explore whether MRCoNS carriage is a risk factor of MRSA colonization in HIV-positive patients.

\section{Materials and Methods}

2.1. Study Design and Population. This cross-sectional study was conducted in a large public HIV clinic between June and August 2017. The public HIV clinic, a HIV outpatient clinic of Guangzhou Eighth People's Hospital, has a population of more than 9000 of HIV-infected patients, about $90 \%$ of all $\mathrm{HIV}$-infected patients in Guangzhou city. All confirmed $\mathrm{HIV}$-infected patients that aged $\geq 18$ years old and agreed with participation were included in the study, while those who had an acute illness were excluded. The study was approved by the Ethics Committee of Guangdong Pharmaceutical University.

2.2. Data Collection. A questionnaire was completed by each enrolled participant after signing an informed consent form. The questionnaire included demographics (age, gender, marital status, and educational level), behavior-related information (smoking status and contact with hay or chaff), details of community-based risk factors (sexual behavior, street drug abuse, and incarceration), medical information (hospitalization, history of influenza, rhinitis, bronchitis, pneumonia, diabetes mellitus, syphilis, and antibiotic use), and HIV-related information (HIV transmission route, CD4 count, and antiretroviral therapy). Nasal samples were collected from both anterior nares of participants using sterile swabs containing sterile saline solution by trained personnel.

\subsection{Isolation and Identification of MRSA and MRCoNS.} Swabs except contacted part were inoculated into $3 \mathrm{~mL}$ enrichment broth (Huankai, Guangzhou, China) containing $7.5 \% \mathrm{NaCl}, 1 \%$ mannitol, $1 \%$ tryptone, and $0.25 \%$ yeast extract and incubated at $36 \pm 1{ }^{\circ} \mathrm{C}$ for 24 hours. A loopful of enrichment broth was plated on mannitol salt agar for another 24 hours of incubation. Colonies morphologically suspicious for Staphylococcus aureus (S. aureus) and CoNS from each mannitol salt agar plate were subcultured to general nutrition agar plates and incubated overnight at
$36 \pm 1^{\circ} \mathrm{C}$. Isolates were identified as $S$. aureus and CoNS based on gram staining, $\beta$-hemolysis test, catalase test, coagulase test, carriage of $16 \mathrm{~S}$ rRNA, and nuc genes. Suspicious isolates were identified as $S$. aureus if they were positive for gram staining, $\beta$-hemolysis test, catalase test, coagulase test, $16 \mathrm{~S}$ rRNA, and nuc genes. Suspicious isolates were identified as CoNS if they were negative for coagulase test and positive for gram staining, $\beta$-hemolysis test, catalase test, and the $16 \mathrm{~S}$ rRNA gene. Detection of $16 \mathrm{~S}$ rRNA and nuc genes was tested by polymerase chain reaction (PCR) assays [10]. S. aureus and CoNS isolates were then, respectively, identified as MRSA and MRCoNS if they were resistant to cefoxitin and positive for the mecA gene [11]. The other S. aureus and CoNS isolates were identified as MSSA and methicillinsusceptible coagulase-negative Staphylococci (MSCoNS), respectively.

2.4. Antimicrobial Susceptibility. Both MRSA and MRCoNS isolates were tested for antibiotic resistance by using Kirby-Bauer disk diffusion method according to the Clinical Laboratory Standards Institute guidelines (CLSI, 2017). Twelve antibiotics were tested, including cefoxitin (FOX: 30ug), penicillin (P: 10unit), erythromycin (E: 15ug), clindamycin (DA: 2ug), tetracycline (TE: 30ug), rifampicin (RD: 5ug), macrodantin (F: 300ug), moxifloxacin (MXF: 5ug), trimethoprim-sulfamethoxazole (SXT: 25ug), teicoplanin (TEC: 30ug), linezolid (LZD: 30ug), and gentamicin (CN: 10ug). Isolates were identified as multidrug resistant (MDR) if they were resistant to $\geq 3$ antibiotic classes [12]. The $S$. aureus isolate ATCC25923 was used for quality control.

2.5. Molecular Characteristics of MRSA. All MRSA isolates were cultured on general nutrition agar plates at $36 \pm 1^{\circ} \mathrm{C}$ for 24 hours. A single bacterial colony was then inoculated into $3 \mathrm{~mL}$ nutritional broth (Huankai, Guangzhou, China) and incubated at $37^{\circ} \mathrm{C}$ with uniformly vigorous shaking for 16 to 18 hours. Finally, the DNA was extracted using Hipura bacterial DNA kit (Magen, Guangzhou, China) based on the manufacturer's instructions.

Genotypic analysis with pulsed-field gel electrophoresis (PFGE) was performed on all confirmed MRSA isolates, including the Panton-Valentine leukocidin gene $(p v l)$ [13], two exfoliating genes (eta and etb) [14], and the toxic shock syndrome toxin gene (tst) [15].

The Staphylococcal cassette chromosome mec (SCCmec) typing of MRSA strains were identified as SCCmec type I, II, III, IV, and V by using multiplex PCR technique [15]. Those isolates which were not SCCmec type I-V were regarded as nontypeable (NT).

The multilocus sequence typing (MLST) PCR assays were performed based on seven housekeeping genes ( $\operatorname{arc} C$, aroE, $g l p F, g m K, p t a, t p i A$, and $y q i L$ ) as previously described [16]. Sequence types were assigned based on the MLST database (http://www.mlst.net). The clonal complex (CC) was determined by using the eBURST algorithm (http://eburst.mlst.net). 
2.6. Statistical Analysis. Frequencies and proportions of MRSA colonization in HIV-infected patients were calculated stratifying by characteristics. Analyses of risk factors of MRSA colonization were examined by nonconditional univariable and multivariable logistic regression models. Chi-square test was conducted to examine the differences of phenotypic characteristics between MRSA and MRCoNS isolates. Two-sided $P$ values $<0.05$ were considered statistically significant. All statistical analyses were performed using Stata version 15.1 (Collage Station, Texas, USA).

\section{Results}

3.1. Prevalence of MRSA and MRCoNS Nasal Colonization. A total of $1026 \mathrm{HIV}$-infected patients were eligible to participate and finally $1001 \mathrm{HIV}$-infected patients were enrolled in this study, including 845 males $(84.42 \%)$ and 156 females (15.58\%). The median age of this population was 35 years (range, 18-81 years). The overall prevalence of S. aureus and MRSA colonization among HIV-infected patients was $25.2 \%$ (253/1001) and $11.9 \%(119 / 1001)$, respectively. Of the 1001 participants, $575(57.4 \%)$ were colonized CoNS isolates and $423(42.3 \%)$ were colonized MRCoNS isolates. And 41 (4.1\%) HIV-infected patients were co-colonized MRSA and MRCoNS isolates.

3.2. Risk Factors for MRSA Colonization. In the nonconditional univariate analyses, there were significant differences of MRSA colonization in terms of educational level, smoking status, contact with hay or chaff, history of upper respiratory tract infection within the previous 6 months, history of syphilis within the previous 6 months, the use of HIV antiretroviral therapy, and the status of CoNS colonization. After adjusting the significant factors listed above by using nonconditional multivariate logistic regression model, we found that contact with hay or chaff in the life (adjusted odds ratio $[\mathrm{aOR}]=1.79,95 \% \mathrm{CI}: 1.02-3.15)$, having a history of syphilis ( $\mathrm{aOR}=1.77,95 \% \mathrm{CI}$ : 1.09-2.90), and suffering from the upper respiratory tract infection within the previous 6 months such as influenza $(\mathrm{aOR}=1.64,95 \% \mathrm{CI}: 1.08-2.49)$ and rhinitis $(\mathrm{aOR}=1.95,95 \% \mathrm{CI}: 1.14-3.32)$ were risk factors of MRSA nasal colonization in HIV-positive persons. Current smoking $(\mathrm{aOR}=0.58,95 \% \mathrm{CI}: 0.37-0.90)$, HIV antiretroviral therapy $(\mathrm{aOR}=0.50,95 \% \mathrm{CI}: 0.27-0.92)$, and colonization of MRCoNS ( $\mathrm{aOR}=0.59,95 \% \mathrm{CI}$ : $0.38-0.91$ ) were protective factors of MRSA colonization in HIVpositive patients (Table 1).

3.3. Antibiotic Resistance. The proportions of antibiotic resistance between MRSA and MRCoNS isolates were significantly different except penicillin, clindamycin, tetracycline, and teicoplanin. Among all MRSA isolates, the most predominant resistant antibiotic was penicillin (89.08\%), while the most predominant resistant antibiotic was also penicillin (93.71\%) among MRCoNS isolates. Note that the proportion of MDR in MRCoNS isolates was significantly higher than that in MRSA (Table 2).
3.4. Molecular Characteristics. Detailed information regarding the molecular characteristics and toxin genes of MRSA is shown in Figure 1. There were 19 CC types and 39 STs in all MRSA isolates in this study. The most predominant ST type among 119 MRSA strains was ST188 (15.1\%, 18/119), followed by ST59 $(11.8 \%, 14 / 119)$ and ST6 $(5.0 \%, 6 / 119)$. The most three predominant CC types among MRSA strains were CC59 (17.6\%, 21/119), CC188 (16.8\%, 20/119), and CC5 (14.29\%, 17/119). In addition, five MRSA isolates were positive for $p v l$ gene and five were positive for tst gene. However, no MRSA isolates were positive for eta and etb genes. Molecular characteristics of MRSA in this study also displayed that $84.03 \%$ of MRSA isolates were NT, followed by SCCmec IV (9.2\%), SCCmec II (3.4\%), SCCmecV (2.5\%), and SCCmec III $(0.8 \%)$.

\section{Discussion}

To the best of our knowledge, this is the first study to explore whether MRCoNS carriage is a risk factor of MRSA colonization. Notably, we found that MRCoNS carriage is a protective factor rather than a risk factor of MRSA colonization. In other words, HIV-infected patients who colonized MRCoNS isolates were less likely to have MRSA colonization.

As for the risk factors of MRSA colonization, we also found that patients who had a history of syphilis were at higher risk for MRSA colonization. This is consistent with a report by Crum-Cianflone et al. [17]. We assumed that this may be caused by lower resistance and immunity in patients with syphilis. It also suggested that MRSA may be transferred during sexual activities and that high-risk behaviors may also increase the risk of MRSA transmission and colonization. Unlike reports of Tilahun et al. [18] and Kyaw et al. [19], we found the history of upper respiratory tract infection rather than lower respiratory tract infection within the previous 6 months was a risk factor of MRSA colonization, which can be associated with the more frequently respiratory episode occurring in $\mathrm{HIV}$-infected population and bacteria colonize in the nasal cavities as the respiratory barrier weakens [20]. Notably, results showed that those who had any contact with hay or chaff were more likely to have MRSA colonization, which was similar to results from Neupane et al. [3]. However, few studies until now investigated the relationships between contact with hay or chaff and MRSA colonization. Similarly, Wang et al. found a significant relationship between smoking status and lower rates of MRSA colonization [21]. In this study, we also found the use of HIV antiretroviral therapy lowered the odds of MRSA colonization as Farley et al. reported [6]. It may suggest that HIV-infected patients using the HIV antiretroviral therapy in primary care clinics could lead to the lower contact with hospitals and then reduce the colonization of MRSA. However, unlike the previous studies $[5,22]$, there was no significant differences between other factors we investigated and MRSA colonization. The potential reasons for the disparity might be the difference in questionnaire design and characteristics of population in different regions. 
TABLe 1: Analyses of risk factors associated with MRSA colonization in HIV-infected patients [OR (95\% CI)].

\begin{tabular}{|c|c|c|c|c|}
\hline Characteristics & Non-SA $(N=748)$ & MRSA $(N=119)$ & OR $(95 \% \mathrm{CI})$ & aOR $(95 \% \mathrm{CI})$ \\
\hline Gender (male) & $631(84.36)$ & $107(89.92)$ & $1.65(0.88-3.10)$ & \\
\hline Age ( $\geq 45$ years) & $202(27.08)$ & $26(21.85)$ & $0.75(0.47-1.19)$ & \\
\hline Marital status (married) & $351(47.18)$ & $45(37.82)$ & $0.68(0.46-1.01)$ & \\
\hline Education (college degree or above) & $261(34.89)$ & $53(44.54)$ & $1.50(1.01-2.22)$ & $1.36(0.90-2.04)$ \\
\hline Been arrested & $41(5.48)$ & $9(7.56)$ & $1.41(0.67-2.98)$ & \\
\hline Current smoker & $288(38.55)$ & $33(27.73)$ & $0.61(0.40-0.94)$ & $0.58(0.37-0.90)$ \\
\hline Street drug abuse* & $39(5.28)$ & $9(7.69)$ & $1.49(0.70-3.17)$ & \\
\hline Contact with hay or chaff* & $71(9.61)$ & $19(15.97)$ & $1.79(1.03-3.09)$ & $1.79(1.02-3.15)$ \\
\hline Hospitalization $*$ & $135(18.10)$ & $14(11.76)$ & $0.60(0.34-1.08)$ & \\
\hline History of influenza* & $398(53.71)$ & $76(64.41)$ & $1.56(1.04-2.33)$ & $1.64(1.08-2.49)$ \\
\hline History of rhinitis* & $79(10.66)$ & $23(19.49)$ & $2.03(1.22-3.38)$ & $1.95(1.14-3.32)$ \\
\hline History of bronchitis* & $26(3.51)$ & $2(1.69)$ & $0.47(0.11-2.02)$ & \\
\hline History of pneumonia* & $27(3.64)$ & $1(0.85)$ & $0.23(0.03-1.68)$ & \\
\hline History of antibiotic use $*$ & $340(46.13)$ & $60(51.28)$ & $1.23(0.83-1.82)$ & \\
\hline History of syphilis* & $111(15.00)$ & $27(22.69)$ & $1.66(1.03-2.67)$ & $1.77(1.09-2.90)$ \\
\hline Diabetes mellitus* & $19(2.55)$ & $1(0.85)$ & $0.33(0.04-2.49)$ & \\
\hline HIV transmission route (sexual) & $498(66.58)$ & $87(73.11)$ & $1.36(0.89-2.10)$ & \\
\hline HIV antiretroviral therapy & $691(92.38)$ & $103(86.55)$ & $0.53(0.29-0.95)$ & $0.50(0.27-0.92)$ \\
\hline CD4 count $(<200$ cells/uL $)$ & $213(28.48)$ & $35(29.41)$ & $1.05(0.68-1.60)$ & \\
\hline \multicolumn{5}{|l|}{ Colonization of CoNS } \\
\hline No & $298(39.84)$ & $65(54.62)$ & Reference & Reference \\
\hline With MSCoNS & $114(15.24)$ & $13(10.92)$ & $0.52(0.28-0.98)$ & $0.52(0.27-1.01)$ \\
\hline With MRCoNS & $336(44.92)$ & $41(34.45)$ & $0.56(0.37-0.85)$ & $0.59(0.38-0.91)$ \\
\hline
\end{tabular}

OR, odds ratio; aOR, adjusted OR; CI, confidence interval; SA, Staphylococcus aureus; MRSA, methicillin-resistant Staphylococcus aureus; MRCoNS, methicillin-resistant coagulase-negative Staphylococci; MSCoNS, methicillin-susceptible coagulase-negative Staphylococci; CoNS, coagulase-negative Staphylococci. Note. $*$ Within the previous 6 months.

TABLE 2: Antibiotic resistance of MRSA and MRCoNS isolates in HIV-infected patients.

\begin{tabular}{|c|c|c|c|c|}
\hline Antibiotic (nonsusceptive) & MRSA $(N=119)$ & MRCoNS $(N=461)$ & $x^{2}$ & $P$ value \\
\hline Penicillin & $106(89.08)$ & $432(93.71)$ & 3.02 & 0.08 \\
\hline Erythromycin & $73(61.34)$ & $336(72.89)$ & 6.06 & 0.01 \\
\hline Clindamycin & $50(42.02)$ & $155(33.62)$ & 2.92 & 0.09 \\
\hline Cefoxitin & $44(36.97)$ & $352(76.36)$ & 67.72 & $<0.01$ \\
\hline Tetracycline & $41(34.45)$ & $161(34.92)$ & 0.01 & 0.92 \\
\hline Trimethoprim-sulfamethoxazole & $24(20.17)$ & $250(54.23)$ & 44.03 & $<0.01$ \\
\hline Moxifloxacin & $28(23.53)$ & $182(39.48)$ & 10.41 & $<0.01$ \\
\hline Macrodantin & $38(31.93)$ & $30(6.51)$ & 59.08 & $<0.01$ \\
\hline Teicoplanin & $39(32.77)$ & $113(24.51)$ & 3.34 & 0.07 \\
\hline Rifampicin & $23(19.33)$ & $54(11.71)$ & 4.76 & 0.03 \\
\hline Gentamicin & $7(5.88)$ & $176(38.18)$ & 45.68 & $<0.01$ \\
\hline Linezolid & $10(8.40)$ & $9(1.95)$ & 12.42 & $<0.01$ \\
\hline MDR & $82(18.06)$ & $372(81.94)$ & 7.73 & $<0.01$ \\
\hline
\end{tabular}

MRSA, methicillin-resistant Staphylococcus aureus; MRCoNS, methicillin-resistant coagulase-negative Staphylococci; MDR, multidrug resistance, resistant to no less than three antibiotic classes.

The prevalence of MRSA nasal colonization in HIVinfected patients was $11.89 \%$, which was higher than that in a Uganda study $(2.41 \%, 4 / 166)$ [7], a Colombian study $(1.06 \%$, 2/283) [23], and a study of Taiwan, China $(3.44 \%, 19 / 553)$ [24], but lower than that in an American study $(15.40 \%, 77 /$ 500) [6] and an Iranian study $(12.78 \%, 23 / 180)$ [25]. The prevalence results demonstrated the high burden of MRSA among HIV-infected patients in China, which might be explained by the overuse of antibiotic in the treatment of complications in HIV-infected patients. It also suggested that this population should pay greater attention to nasal hygiene to avoid person-to-person transmission of MRSA isolates. Compared with the previous studies, the differences in prevalence of MRSA might be affected by geological factors, limited sampling locations, and the varying techniques for isolation and identification of bacterial strains.

Among all MRSA isolates, high drug resistance was observed in penicillin, erythromycin, and clindamycin, which is similar to observed studies $[4,26,27]$. In this study, we believe the MRSA and MRCoNS isolates may show differences in all antibiotic resistance. However, opposite to our hypothesis, the antibiotic resistance did not show a statistical difference in penicillin, clindamycin, tetracycline, and teicoplanin. It suggested that there were similarities on 


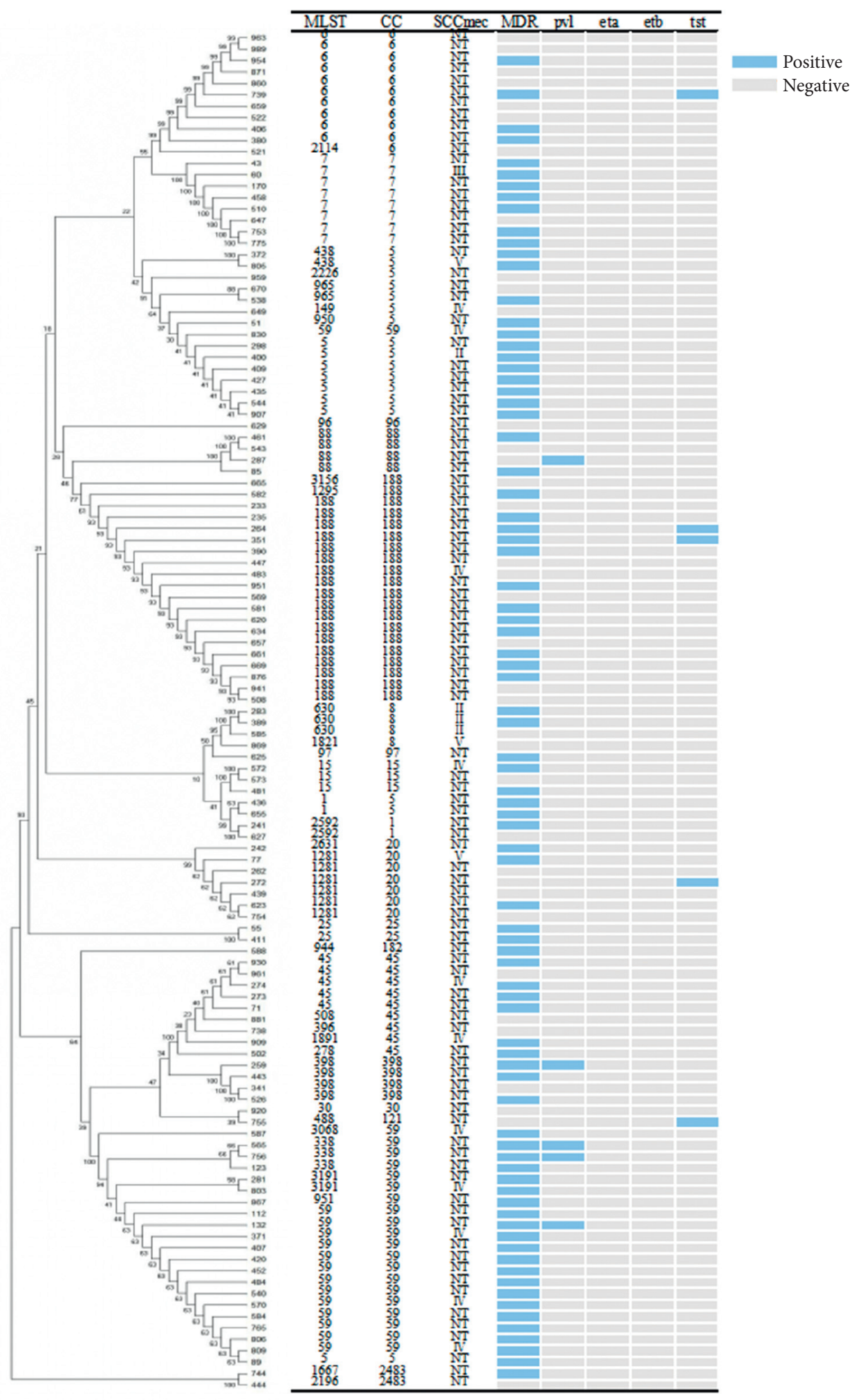

FIGURE 1: The sequence type and detailed information of MRSA isolates. CC, clonal complex; MLST, multilocus sequence typing; SCCmec, staphylococcal cassette chromosome mec; NT, nontypeable; MRSA, methicillin-resistant Staphylococcus aureus; MDR, multidrug resistance, resistant to no less than three antibiotic classes; $p v l$, Panton-Valentine leukocidin; eta, exfoliative toxin A; etb, exfoliative toxin B; tst, toxic shock syndrome toxin.

the resistance to antibiotics listed above between MRSA and MRCoNS isolates. We supposed the reason was that co- colonization of MRSA and MRCoNS isolates in humans may lead to horizontal cross-propagation of resistance genes as 
Al-Bakri et al. reported [9]. Further studies are needed to explore the relationship of antimicrobial resistance between MRSA and MRCoNS strains at molecular level. The findings of multidrug resistance of MRSA and MRCoNS isolates in this study were also noteworthy. A significant difference was found between them, in which the multidrug resistant rate in MRCoNS (81.94\%) was much higher than that in MRSA (18.06\%). This result illustrated that MRCoNS may play an important role in drug resistance. Not only MRSA but also MRCoNS needs to pay more attention to surveillance and monitoring programs.

It was well known that Panton-Valentine leukocidin ( $p v l)$, a virulent factor encoding toxin, may have a significant influence in some serious $S$. aureus infections, such as severe skin and soft tissue infection and necrotizing pneumonia [28]. We found five $(4.20 \%, 5 / 119)$ MRSA isolates were positive for $p v l$ toxin gene in this study, which was much lower than a study in Japan (33.33\%) [29]. Regarding the virulence genes, another five (4.20\%) MRSA isolates were found to be positive for tst gene, which was much lower than a study reported among hospital patients $(11.60 \%)$ [30]. We speculated that the low carrying rate of $p v l$ and tst genes in the study might be related to the unique characteristics of each population and regional disparity.

A total of 39 STs and 19 CC types of all MRSA isolates were found in our study. Results showed that ST188 was the predominant ST types of MRSA isolates among HIV-infected patients in mainland, China, and ST59 in Taiwan, China [24]. The dominant STs (ST188, ST59 and ST6) in this study were consistent with other community-based populations [31-33], but some other STs were also previously reported in hospital patients (ST338 and ST1) [34-36] and animals (ST398 and ST1) [37-39], which might indicate the cross transmission of isolates in different sources. CC59, the most predominant clonal complex types of MRSA in our study, is the predominant community-associated MRSA clone in Asia [40]. CC5, the third major CC types of MRSA in this study, was reported to be the main CC type of HA-MRSA [41]. The distribution of CC types of MRSA indicated that HA-MRSA strains were gradually infiltrating into the community populations. Based on the distribution of SCCmec typing, we found most MRSA isolates in this study were nontypeable, followed by SCCmec IV and SCCmec III. Both HA-MRSA and CA-MRSA were detected in this HIV-positive population, which also suggested that HAMRSA strains were infiltrating into the community groups.

As the first study to explore whether MRCoNS carriage is a risk factor of MRSA colonization, some limitations should be considered when interpreting the results. Firstly, we can only describe associations between influencing factors and MRSA colonization by using a cross-sectional design, not a causal conclusion. Secondly, we administered the sampling at only one site, which may underestimate the prevalence of MRSA colonization. Thirdly, colonization was only assessed on the day of enrollment. Evaluation of durability of colonization may be useful to fully understand MRSA colonization dynamics.

\section{Conclusions}

HIV-infected patients remain a highly vulnerable population for MRSA colonization, and MRCoNS colonization is a protective factor for MRSA colonization. Moreover, we found similar antibiotic patterns between MRSA and MRCoNS. Further studies of relationship between MRSA and MRCoNS explored at molecular level are needed to examine our hypothesis. Our findings suggested that it is still necessary to pay more attention to MRSA colonization and its antibiotic pattern as well as MRCoNS.

\section{Data Availability}

The data used to support the findings of this study are available from the corresponding author upon request.

\section{Conflicts of Interest}

The authors declare that they have no conflicts of interest.

\section{Acknowledgments}

This research was funded by Guangdong Science and Technology Plan Project (Grant no. 2014A020213013) and National Natural Science Foundation of China (Grant nos. 81973069 and 81602901).

\section{References}

[1] A. Hassoun, P. K. Linden, and B. Friedman, "Incidence, prevalence, and management of MRSA bacteremia across patient populations-a review of recent developments in MRSA management and treatment," Critical Care, vol. 21, no. 1, p. $211,2017$.

[2] S. Lakhundi and K. Zhang, "Methicillin-resistant Staphylococcus aureus: molecular characterization, evolution, and epidemiology," Clinical Microbiology Reviews, vol. 31, no. 4, Article ID e00020, 2018.

[3] K. Neupane, B. Rayamajhee, and J. Acharya, "Comparison of nasal colonization of methicillin-resistant Staphylococcus aureus in HIV-infected and non-HIV patients attending the national public health laboratory of Central Nepal," Canadian Journal of Infectious Diseases and Medical Microbiology, vol. 2018, Article ID 4508757, 2018.

[4] C. Regina Pedrosa Soares, C. R. de Lira, and M. A. H. Cunha, "Prevalence of nasal colonization by methicillin-resistant Staphylococcus aureus in outpatients living with HIV/AIDS in a Referential Hospital of the Northeast of Brazil," BMC Research Notes, vol. 11, no. 1, p. 794, 2018.

[5] P. Sabbagh, S. M. Riahi, H. R. Gamble, and A. Rostami, "The global and regional prevalence, burden, and risk factors for methicillin-resistant Staphylococcus aureus colonization in HIV-infected people: a systematic review and meta-analysis," American Journal of Infection Control, vol. 47, no. 3, pp. 323-333, 2019.

[6] J. E. Farley, M. J. Hayat, P. L. Sacamano, T. Ross, and K. Carroll, "Prevalence and risk factors for methicillin-resistant Staphylococcus aureus in an HIV-positive cohort," American Journal of Infection Control, vol. 43, no. 4, pp. 329-335, 2015.

[7] L. M. Bebell, A. Ayebare, Y. Boum et al., "Prevalence and correlates of MRSA and MSSA nasal carriage at a Ugandan regional referral hospital," The Journal of Antimicrobial Chemotherapy, vol. 72, no. 3, pp. 888-892, 2017.

[8] A. Martins, R. S. de Lourdes, and M. Cunha, "Methicillin resistance inStaphylococcus aureusand coagulase-negative 
staphylococci: epidemiological and molecular aspects," $M i$ crobiology and Immunology, vol. 51, no. 9, pp. 787-795, 2007.

[9] A. G. Al-Bakri, H. Al-Hadithi, V. Kasabri, G. Othman, A. Kriegeskorte, and K. Becker, "The epidemiology and molecular characterization of methicillin-resistant staphylococci sampled from a healthy Jordanian population," Epidemiology and Infection, vol. 141, no. 11, pp. 2384-2391, 2013.

[10] H.-Y. Wang, S. Kim, J. Kim, S.-D. Park, Y. Uh, and H. Lee, "Multiplex real-time PCR assay for rapid detection of methicillin-resistant staphylococci directly from positive blood cultures," Journal of Clinical Microbiology, vol. 52, no. 6 , pp. 1911-1920, 2014.

[11] A. Huletsky, R. Giroux, V. Rossbach et al., "New real-time PCR assay for rapid detection of methicillin- resistant Staphylococcus aureus directly from specimens containing a mixture of staphylococci," Journal of Clinical Microbiology, vol. 42, no. 5, pp. 1875-1884, 2004.

[12] A.-P. Magiorakos, A. Srinivasan, R. B. Carey et al., "Multidrug-resistant, extensively drug-resistant and pandrug-resistant bacteria: an international expert proposal for interim standard definitions for acquired resistance," Clinical Microbiology and Infection, vol. 18, no. 3, pp. 268-281, 2012.

[13] N. Abimanyu, A. Krishnan, S. Murugesan, G. K. Subramanian, S. Gurumurthy, and P. Krishnan, "Use of triplex PCR for rapid detection of PVL and differentiation of MRSA from methicillin resistant coagulase negative staphylococci," Journal of Clinical and Diagnostic Research: JCDR, vol. 7, no. 2, pp. 215-218, 2013.

[14] D. Shi, S. Ishii, T. Sato et al., "Staphylococcal scalded skin syndrome in an extremely low-birth-weight neonate: molecular characterization and rapid detection by multiplex and real-time PCR of methicillin-resistant Staphylococcus aureus," Pediatrics International, vol. 53, no. 2, pp. 211-217, 2011.

[15] M. Motoshima, K. Yanagihara, Y. Morinaga et al., "Genetic diagnosis of community-acquired MRSA: a multiplex realtime PCR method for Staphylococcal cassette chromosome mec typing and detecting toxin genes," The Tohoku Journal of Experimental Medicine, vol. 220, no. 2, pp. 165-170, 2010.

[16] Y.-T. Lee, D.-B. Lin, W.-Y. Wang et al., "First identification of methicillin-resistant Staphylococcus aureus MLST types ST5 and ST45 and SCCmec types IV and Vt by multiplex PCR during an outbreak in a respiratory care ward in central Taiwan," Diagnostic Microbiology and Infectious Disease, vol. 70, no. 2, pp. 175-182, 2011.

[17] N. F. Crum-Cianflone, A. A. Burgi, and B. R. Hale, "Increasing rates of community-acquired methicillin-resistant Staphylococcus aureus infections among HIV-infected persons," International Journal of STD \& AIDS, vol. 18, no. 8, pp. 521-526, 2007.

[18] B. Tilahun, A. C. Faust, P. McCorstin, and A. Ortegon, "Nasal colonization and lower respiratory tract infections with methicillin-resistant Staphylococcus aureus," American Journal of Critical Care, vol. 24, no. 1, pp. 8-12, 2015.

[19] W. Kyaw, L. Lee, W. Siong, A. C. Ping, B. Ang, and Y. Leo, "Prevalence of and risk factors for MRSA colonization in HIV-positive outpatients in Singapore," AIDS Research and Therapy, vol. 9, no. 1, p. 33, 2012.

[20] R. Miller, "HIV-associated respiratory diseases," The Lancet, vol. 348, no. 9023, pp. 307-312, 1996.

[21] J.-T. Wang, C.-H. Liao, C.-T. Fang et al., "Prevalence of and risk factors for colonization by methicillin-resistant Staphylococcus aureus among adults in community settings in Taiwan," Journal of Clinical Microbiology, vol. 47, no. 9, pp. 2957-2963, 2009.
[22] K. J. Popovich, K. Y. Smith, T. Khawcharoenporn et al., "Community-associated methicillin-resistant Staphylococcus aureus colonization in high-risk groups of HIV-infected patients," Clinical Infectious Diseases, vol. 54, no. 9, pp. 1296-1303, 2012.

[23] M. Hidalgo, L. P. Carvajal, and S. Rincón, "Methicillin-resistant Staphylococcus aureus USA300 Latin American variant in patients undergoing hemodialysis and HIV infected in a hospital in bogotá, Colombia," PLoS One, vol. 10, no. 10, Article ID e0140748, 2015.

[24] Y. Y. Hsu, D. Wu, and C. C. Hung, "Methicillin-resistant Staphylococcus aureus nasal colonization among HIV-infected patients in Taiwan: prevalence, molecular characteristics and associated factors with nasal carriage," $B M C$ Infectious Diseases, vol. 20, no. 1, p. 254, 2020.

[25] P. M. Hassanzadeh, Y. M. Hassanzadeh, and J. P. Mardaneh, "Isolation of methicillin-resistant Staphylococcus aureus (MRSA) from HIV patients referring to HIV referral center, Shiraz, Iran, 2011-2012," Iranian Journal of Medical Sciences, vol. 40, no. 6, pp. 526-530, 2015.

[26] M. T. Lemma, Y. Zenebe, and B. Tulu, "Methicillin resistant Staphylococcus aureus among HIV infected pediatric patients in northwest Ethiopia: carriage rates and antibiotic co-resistance profiles," PLoS One, vol. 10, no. 9, Article ID e0137254, 2015.

[27] M. J. Groome, W. C. Albrich, J. Wadula, M. Khoosal, and S. A. Madhi, "Community-onsetStaphylococcus aureusbacteraemia in hospitalised African children: high incidence in HIV-infected children and high prevalence of multidrug resistance," Paediatrics and International Child Health, vol. 32, no. 3, pp. 140-146, 2012.

[28] C. Zhang, Y. Guo, and X. Chu, "In Vitro generation of panton-valentine leukocidin (PVL) in clinical MethicillinResistant Staphylococcus aureus (MRSA) and its correlation with PVL variant, clonal complex, infection type," Science Reports, vol. 8, no. 1, p. 7696, 2018.

[29] K. Hirota, D. Watanabe, and Y. Koizumi, "Observational study of skin and soft-tissue Staphylococcus aureus infection in patients infected with HIV-1 and epidemics of pantonvalentine leucocidin-positive community-acquired MRSA infection in Osaka, Japan," Journal of Infection and Chemotherapy, vol. 26, no. 12, pp. 1254-1259, 2020.

[30] M. Motamedifar, H. S. Ebrahim-Saraie, S. M. H. Alfatemi, M. Zalipour, M. Kaveh, and H. Khoshkharam-Roodmajani, "Frequency of the toxic shock syndrome toxin-1 gene in methicillin-susceptible and -resistant Staphylococcus aureus isolates from teaching hospitals in Shiraz, Iran," Revista da Sociedade Brasileira de Medicina Tropical, vol. 48, no. 1, pp. 90-93, 2015.

[31] M. Goudarzi, S. S. Seyedjavadi, M. J. Nasiri, H. Goudarzi, R. Sajadi Nia, and H. Dabiri, "Molecular characteristics of methicillin-resistant Staphylococcus aureus (MRSA) strains isolated from patients with bacteremia based on MLST, SCCmec, spa, and agr locus types analysis," Microbial Pathogenesis, vol. 104, pp. 328-335, 2017.

[32] J. Lin, J. Liang, T. Zhang, C. Bai, J. Ye, and Z. Yao, "Doseresponse associations of methicillin-resistant Staphylococcus aureus between school environmental contamination and nasal carriage by elementary students," Infection and Drug Resistance, vol. 11, pp. 773-782, 2018.

[33] J. Lin, C. Wu, Q. Ou et al., "Nasal colonization of Staphylococcus aureus colonal complex 5: prevalence, influencing factors, and phenotypic and molecular characteristics in 
pregnant Chinese women," American Journal of Infection Control, vol. 45, no. 10, pp. 1106-1110, 2017.

[34] J. Lin, C. a. Wu, C. Yan et al., "A prospective cohort study of Staphylococcus aureus and methicillin-resistant Staphylococcus aureus carriage in neonates: the role of maternal carriage and phenotypic and molecular characteristics," Infection and Drug Resistance, vol. 11, pp. 555-565, 2018.

[35] Y.-T. Tang, R. Cao, N. Xiao et al., "Molecular epidemiology and antimicrobial susceptibility of methicillin-resistant Staphylococcus aureus isolates in Xiangyang, China," Journal of Global Antimicrobial Resistance, vol. 12, pp. 31-36, 2018.

[36] G. Omuse, K. N. Van Zyl, and K. Hoek, "Molecular characterization of Staphylococcus aureus isolates from various healthcare institutions in Nairobi, Kenya: a cross sectional study," Annals of Clinical Microbiology and Antimicrobials, vol. 15, no. 1, p. 51, 2016.

[37] T. Conceição, H. de Lencastre, and M. Aires-de-Sousa, "Frequent isolation of methicillin resistant Staphylococcus aureus (MRSA) ST398 among healthy pigs in Portugal," PLoS One, vol. 12, no. 4, Article ID e0175340, 2017.

[38] B. Schauer, R. Krametter-Frötscher, F. Knauer et al., "Diversity of methicillin-resistant Staphylococcus aureus (MRSA) isolated from Austrian ruminants and New World camelids," Veterinary Microbiology, vol. 215, pp. 77-82, 2018.

[39] J. R. Wipf and V. Perreten, "Methicillin-resistant Staphylococcus aureus isolated from dogs and cats in Switzerland," Schweiz Arch Tierheilkd, vol. 158, no. 6, pp. 443-450, 2016.

[40] C. Glasner, G. Pluister, H. Westh et al., "Staphylococcus aureus spa type t437: identification of the most dominant community-associated clone from Asia across Europe," Clinical Microbiology and Infection, vol. 21, no. 2, pp. e1-e163, 2015.

[41] M. Otto, "MRSA virulence and spread," Cellular Microbiology, vol. 14, no. 10, pp. 1513-1521, 2012. 